\title{
Impacto de ação educativa na manutenção do decúbito elevado como medida preventiva de pneumonia associada à ventilação mecânica em Unidade de Terapia Intensiva
}

\author{
Impact of educational action in maintaining high decubitus as preventive \\ measure of ventilator-associated pneumonia in the Intensive Care Unit
}

\author{
Fabiana Petruske Niyama Mansano ${ }^{1}$, Renata Aparecida Belei ${ }^{1}$, Léo Augusto da Silva Vinci², Bruna Luiza Dutra de Melo³ \\ Lucienne Tibery Queiroz Cardoso², Joseani Coelho Pascual Garcia1', Claudia Maria Dantas de Maio Carrilho', \\ Gabriela Machado Ezaias², Cintia Magalhães Carvalho Grion² \\ ${ }^{1}$ Hospital Universitário de Londrina (HU) - Londrina (PR), Brasil. \\ 2Universidade Estadual de Londrina (UEL) - Londrina (PR), Brasil. \\ ${ }^{3}$ Secretaria de Estado da Saúde do Paraná (SESA) - Londrina (PR), Brasi
}

DOI: http://dx.doi.org/10.7322/abcshs.v42i1.945

\begin{abstract}
RESUMO
Introdução: A pneumonia adquirida na Unidade de Terapia Intensiva (UTI) ocasiona o prolongamento da hospitalização e impacta na mortalidade. Intervenções educativas com profissionais de saúde são estratégias de prevenção relevantes nas infecções relacionadas à assistência à saúde. Objetivo: Avaliar o resultado de uma ação educativa na adesão à manutenção do decúbito elevado, acima de 30 graus, como medida para prevenção de pneumonia associada à ventilação mecânica. Métodos: Estudo quase experimental do tipo antes e depois desenvolvido na UTI do Hospital Universitário de Londrina, no período de março e junho de 2010, dividido em três fases (pré-intervenção, intervenção e pós-intervenção). Durante o período de estudo, foram treinados 49 profissionais. Foram realizadas observações diretas da altura do decúbito antes e após o treinamento e coletados dados clínicos dos pacientes admitidos na UTI. A adesão à recomendação de manter o decúbito elevado foi definida como a manutenção de decúbito acima de 30 graus para cada observação. Resultados: A média de angulação do decúbito apresentou aumento significativo do período pré-intervenção $(27,85$ $\pm 6,76$ graus) para o primeiro mês pós-intervenção $(30,70 \pm 8,18$ graus; $p<0,001$ ), no entanto esses valores não persistiram no terceiro mês de observação pós-intervenção (29,46 $\pm 6,19$ graus). Conclusão: A ação educativa mostrou-se eficaz a curto prazo, na adesão à elevação do decúbito em ambiente hospitalar, porém essa adesão sofreu redução progressiva, demonstrando a necessidade de uma intervenção continuada para manutenção dos resultados.
\end{abstract}

Palavra-chave: pneumonia associada à ventilação mecânica; infecção hospitalar; /prevenção \& controle; educação em saúde.

\begin{abstract}
Introduction: Intensive Care Unit (ICU) acquired pneumonia prolongs hospitalization and influences mortality rates. Educational interventions with health professionals are relevant preventive strategies for healthcare-associated infections. Objective: To evaluate the result of an educational activity on adherence to the maintenance of a high decubitus, above 30 degrees, as a measure for preventing ventilator-associated pneumonia. Methods: Quasi-experimental study with a pretestposttest design conducted in the ICU of the University Hospital of Londrina from March to June 2010, divided in three periods (preintervention, intervention and post-intervention). During study period, 49 health professionals were trained. Direct observations of the decubitus' height were performed before and after training and clinical data of patients admitted to the ICU were collected. Adherence to the recommendation of high decubitus was defined as maintenance of decubitus above 30 degrees in each observation. Results: There was an increase in the mean of decubitus' angle from pre-intervention period (27.85 \pm 6.76 degrees) to post-intervention $(30.70 \pm 8.18$ degrees; $p<0.001)$, although this difference did not persisted in the third month postintervention observation (29.46 \pm 6.19 degrees). Conclusion: The educational activity was transiently effective in increasing adherence to maintain high decubitus during hospitalization, but the adherence decreased progressively, showing the need for a continuous intervention to maintain results.
\end{abstract}

Keywords: pneumonia, ventilator-associated; cross infection; / prevention \& control; health education. 


\section{INTRODUÇÃO}

A pneumonia é uma das infecções relacionadas à assistência à saúde mais frequente entre os pacientes graves com uso de ventilação mecânica, podendo atingir até $28 \%$ desses pacientes nas Unidades de Terapia Intensiva (UTI) ${ }^{1}$. Várias estratégias preventivas têm demonstrado sucesso na redução da incidência dessa infecção, porém ela permanece apresentando grande impacto no prognóstico de pacientes graves e resultando em aumento nos custos relacionados à saúde 2 .

Vários são os fatores de risco para o desenvolvimento de pneumonia nosocomial na UTI, como: idade avançada, uso de ventilação mecânica invasiva, doença aguda ou crônica grave, hospitalização prolongada, falha em medidas para o controle das infecções relacionadas à assistência em saúde, uso de bloqueadores neuromusculares, antibióticos, antiácidos, intervenções cirúrgicas neurológicas, posição supina no leito, dieta enteral, sondas enterais, entre outros ${ }^{3,4}$

Os episódios de aspiração são mais frequentes quando o paciente é posicionado em posição supina. Estudos sugerem que o posicionamento do decúbito do leito elevado, entre 30 e 45 graus, reduz a frequência e o risco da pneumonia principalmente em pacientes que recebem nutrição enteral, naqueles sob ventilação mecânica prolongada e rebaixamento da consciência, uma vez que minimiza o refluxo do conteúdo do estômago para o pulmão ${ }^{5,6}$.

São muitos os fatores relacionados à inadequação dessa prática, porém a falta de clareza sobre a importância dessa atividade na prevenção de pneumonia constitui-se no principal fator de risco para a prática inadequada. Como forma de reverter essa situação, a educação mostra-se como uma das estratégias mais relevantes e, por essa razão, faz-se necessário promover intervenções educativas com os profissionais da saúde para que estejam em alerta quanto à gravidade das pneumonias hospitalares e à necessidade de se implantar e supervisionar diariamente a manutenção do decúbito elevado.

O objetivo principal deste estudo foi realizar uma ação educativa para manutenção do decúbito elevado durante 24 horas, como medida preventiva de infecção em UTI. Como objetivo secundário, foi avaliado o impacto da ação educativa na frequência de pneumonia associada à ventilação mecânica.

\section{MÉTODOS}

Trata-se de um estudo quase experimental, do tipo antes e depois. As intervenções do estudo foram práticas educativas envolvendo profissionais de saúde em UTI, com tempo mínimo de 12 meses de atuação na unidade. Foi considerada população do estudo os profissionais de saúde que atuam em UTI. A amostragem foi realizada incluindo consecutivamente todos os profissionais de saúde que estavam no local durante o período do estudo. Foram excluídos os profissionais ausentes ou aqueles que se recusaram a participar. Como avaliação do efeito das práticas educativas, foram analisadas a angulação do decúbito do leito de
UTI e a frequência de pneumonia associada à ventilação mecânica antes e após a intervenção. O local de estudo foi o Hospital Universitário da Universidade Estadual de Londrina (UEL), caracterizado por ser público, terciário, de grande porte, com 330 leitos. A UTI para adultos do Hospital Universitário dispõe de 17 leitos, distribuídos em 2 unidades de 10 e 7 leitos, e atende pacientes clínicos e cirúrgicos.

A pesquisa foi desenvolvida de acordo com os princípios éticos contidos na declaração de Helsinki e foi aprovada pelo Comitê de Ética em Pesquisa local, parecer no $191 / 2008$. O cronograma do estudo incluiu três períodos: observação pré-intervenção (início de março de 2010), intervenção (março de 2010) e observação pós-intervenção (abril a junho de 2010).

\section{Período de observação pré-intervenção}

Durante o período de observação pré-intervenção (10 dias), foram realizadas visitas diárias a todos os leitos da UTI adulto do Hospital Universitário. As visitas ocorreram em um dos três turnos: matutino (7 às 13 horas), vespertino (13 às 19 horas) ou noturno (19 às 24 horas). Os turnos foram sorteados previamente para garantir que a avaliação fosse uniforme nos três. As informações sobre o horário das visitas ficaram restritas aos pesquisadores.

Durante as visitas foram coletados dados sobre a mensuração da angulação do decúbito por meio de um transferidor de grau de 0 a 180 graus com régua. $\mathrm{O}$ ângulo compreendeu a medida entre zero grau, presente na base do transferidor alinhado à lateral inferior da base da cabeceira da cama, e o valor encontrado na parte inferior da régua, alinhada à parte superior articulada do leito, que sustenta o colchão.

As camas utilizadas pelos pacientes no período de estudo foram camas com duas ou três manivelas articuláveis para movimentos fowler e posição de Trendelemburg, com grades em tubos 7/8. Os colchões eram de espuma de poliuretano, com densidade 33 e dimensões de 188 × 88 × $10 \mathrm{~cm}$ de altura. As capas dos colchões eram de courvin soldadas eletronicamente, com zíper e respiros. Além do ângulo do decúbito, foram anotadas presença de sonda nasogástrica e administração de dieta enteral, fatores que aumentam o risco de broncoaspiração. Também foi observado se o paciente fazia uso de mais de um travesseiro na região da cabeça ou de outro objeto com a mesma função.

\section{Período de intervenção}

Foi realizada uma ação educativa com a equipe de profissionais que atua na UTI adulto, incluindo médicos, enfermagem, fisioterapia e alunos de graduação e pós-graduação. A abordagem seguiu um roteiro pré-elaborado com pontos relevantes sobre a manutenção do decúbito elevado e cuidados antes, durante e após a administração de dieta enteral. Nessa etapa, os participantes foram orientados sobre a finalidade da pesquisa, tendo sido realizada uma discussão com base no roteiro de treinamento preestabelecido e exposição de cartazes com figuras demonstrando as diferenças nas taxas de aspiração do conteúdo gástrico para pacientes em 
decúbito deitado e semissentado. Esses cartazes também foram afixados posteriormente à cabeceira de cada leito com o objetivo de alertar os profissionais quanto à angulação ideal do decúbito.

Os pesquisadores realizaram o treinamento no horário de trabalho, no ambiente da UTI. Foram treinados 49 profissionais no período de intervenção. Após o convite e a aceitação, o profissional era conduzido individualmente ao Posto de Enfermagem, onde recebia o termo de consentimento, assinava o caderno de registros de treinamentos da Comissão de Controle de Infecção Hospitalar (CCIH) e, com o pesquisador, lia o roteiro de treinamento, discutindo as dúvidas e experiências. O cartaz com a imagem da aspiração nas posições sentada e deitada era mostrado, sendo explicada a diferença entre cada posição e demonstrado o ângulo correto de 30 graus. Não houve recusa em participar da ação educativa pelos profissionais e estudantes convidados. Os motivos para não receber o treinamento foram absenteísmo previsto por férias ou licença em todos os casos.

Vale ressaltar que, nos dois primeiros meses do período de observação pós-intervenção, os cartazes com as ilustrações da altura de 30 graus do decúbito ficaram afixados e foram retirados apenas no terceiro mês desse período.

\section{Período de observação pós-intervenção}

Nos três meses seguintes ao treinamento, foram realizadas as visitas pós-intervenção para avaliar o comportamento dos participantes diante da adesão das medidas de prevenção de pneumonia associada à ventilação mecânica. Essa avaliação foi realizada uma vez ao dia em um dos três turnos, também de forma sorteada, para que a avaliação fosse uniforme nos três turnos: matutino (7 às 13 horas), vespertino (13 às 19 horas) ou noturno (19 às 24 horas).

Durante o período de estudo, foram coletados dados sobre os pacientes internados na UTI. As variáveis coletadas foram dados demográficos (sexo e idade), diagnóstico de admissão, diagnóstico de pneumonia adquirida na UTI, necessidade de ventilação mecânica e uso de nutrição enteral. Também foram colhidos dados para o cálculo do escore de gravidade Acute Physiology and Chronic Health Evaluation II (APACHE II) e escore de disfunção orgânica Sequential Organ Failure Assessment (SOFA). Os pacientes receberam acompanhamento durante a internação na UTI até a alta, e foi anotado o desfecho da UTI.

O escore SOFA foi calculado nas primeiras 24 horas após a admissão do paciente na UTI, sendo utilizado o pior valor para cada variável do período. Os sistemas orgânicos considerados foram: respiratório, cardiovascular, renal, hematológico, hepático e neurológico. Cada um dos sistemas foi pontuado numa escala de $0-4$, de acordo com a gravidade da disfunção, resultando um escore total de $0-24^{7}$.

A gravidade da doença foi avaliada pelo escore APACHE II, coletado nas primeiras 24 horas de internação, e a presença de doença crônica foi definida de acordo com os critérios descritos por esse escore ${ }^{8}$.

O diagnóstico de pneumonia baseou-se em uma combinação de critérios clínicos, laboratoriais e radiológicos, segundo recomendações do Centers for Disease Control and Prevention $(\mathrm{CDC})^{9}$. Pneumonia associada à ventilação mecânica foi definida como processo infeccioso do parênquima pulmonar que ocorreu após 48 horas da admissão ou alta da UTI, ou associado ao procedimento invasivo que ocorreu durante a permanência na UTI. A adesão à recomendação de manter o decúbito elevado foi definida como a manutenção de decúbito igual ou acima de 30 graus para cada observação ${ }^{5}$.

\section{Análise estatística}

Cálculo do tamanho amostral: para detectar diferença de 4 graus na medida da angulação do decúbito entre o período pré e pós-intervenção, adotando média inicial de 25 graus, desvio padrão de 5, poder de $80 \%$ e significância de $95 \%$, são necessárias 26 observações em cada período.

Para análise estatística, as variáveis contínuas são apresentadas como média e desvio padrão (para o caso de distribuição normal) e como mediana e interquartil (se distribuição não normal). As variáveis categóricas estão expressas como proporção. Estatística descritiva foi utilizada para a apresentação de todas as variáveis relevantes. Para estimar e testar as hipóteses sobre as médias e as variâncias, foi aplicado teste $t$ de Student ou Mann Whitney de acordo com a distribuição dos dados. As frequências das variáveis qualitativas ou categóricas foram avaliadas pelo teste de $\chi^{2}$. O nível de significância adotado foi de $5 \%(\mathrm{a}=0,05)$.

\section{RESULTADOS}

Foi observado aumento da média de angulação do decúbito entre o período pré-intervenção $(27,85 \pm 6,76$ graus $)$ e o primeiro mês pós-intervenção $(30,71 \pm 9,06$ graus; $\mathrm{p}<0,001)$, com redução progressiva dessa média nos períodos subsequentes. Ao analisarmos a proporção de observações em que o decúbito se encontrava em adesão às recomendações, observou-se aumento significativo de 44,8 para $62,1 \%(\mathrm{p}=0,008)$ no primeiro mês pós-intervenção e redução gradual posterior (Tabela 1). Não foi observado o uso de

Tabela 1: Descrição dos resultados das medidas da angulação da cabeceira do leito nos períodos de estudo

\begin{tabular}{|l|c|c|c|c|} 
& $\begin{array}{c}\text { Pré-intervenção } \\
(\mathbf{n}=\mathbf{9 6})\end{array}$ & $\begin{array}{c}\text { Primeiro mês } \\
\text { pós-intervenção } \\
(\mathbf{n}=\mathbf{1 9 0})\end{array}$ & $\begin{array}{c}\text { Segundo mês } \\
\text { pós-intervenção } \\
(\mathbf{n}=\mathbf{1 9 4})\end{array}$ & $\begin{array}{c}\text { Terceiro mês } \\
\text { pós-intervenção } \\
(\mathbf{n}=\mathbf{9 8})\end{array}$ \\
\hline Angulação da cabeceira (graus) $^{*}$ & $27,85 \pm 6,76$ & $30,71 \pm 9,06$ & $31,33 \pm 8,11$ & $29,46 \pm 6,19$ \\
\hline Adesão à recomendação $^{\dagger}$ & $43(44,8 \%)$ & $118(62,1 \%)$ & $115(59,3 \%)$ & $51(52,0 \%)$ \\
\hline
\end{tabular}

*resultados expressos em média e desvio padrão; tresultados expressos em frequência absoluta e proporção. 
mais de um travesseiro na região da cabeça do paciente, ou objeto com a mesma finalidade, apesar de algumas camas não alcançarem a angulação igual ou superior a 30 graus, mesmo com a manivela na posição máxima.

Durante o período do estudo, 170 pacientes foram admitidos na UTI, tinham em média $57,47 \pm 20,53$ anos e $58,8 \%$ eram do sexo masculino. Os diagnósticos de admissões mais frequentes foram sepse $62 / 170$ (36,5\%); hemorragia cerebral 18/170 (10,6\%); pós-operatório de neurocirurgia 15/170 (8,8\%); pós-operatório de trauma, insuficiência respiratória em paciente cirúrgico e pós-parada cardiorrespiratória 8/170 (4,7\%) cada; pós-operatório de cirurgia cardíaca $6 / 170$ (3,5\%); pós-operatório de cirurgia do aparelho digestivo, pós-operatório de cirurgia do aparelho respiratório e pós-operatório de cirurgia vascular 5/170 (2,9\%); trauma de crânio 4/170 (2,4\%); e outros 26/170 (15,3\%).

Com relação aos escores de gravidade, esses pacientes apresentavam média de APACHE II de 19,43 \pm 9,21 e média de escore de disfunção orgânica SOFA de $6,45 \pm 3,74$. Com relação ao uso de intervenções terapêuticas, $109 / 170$ pacientes $(64,1 \%)$ estavam em ventilação mecânica invasiva no dia da admissão na UTI e 117/170 $(68,8 \%)$ necessitaram de ventilação mecânica em algum momento durante a permanência na UTI. Os pacientes ficaram em média 7,62 \pm 10,53 dias em ventilação mecânica e 103/170 pacientes $(60,6 \%)$ fizeram uso de suporte nutricional enteral. O diagnóstico de pneumonia adquirida na UTI foi feito em 56/170 (32,9\%) pacientes e a taxa de mortalidade geral dos pacientes no período de estudo foi $36,5 \%$.

Comparando-se os dados clínicos dos pacientes nos períodos pré e pós-intervenção, observou-se que os pacientes tinham características demográficas e valores de escores de gravidade semelhantes nesses dois períodos. Não foi detectada redução na frequência de pneumonia ou na taxa de mortalidade (Tabela 2).

\section{DISCUSSÃO}

O principal achado deste estudo foi um aumento transitório da adesão às recomendações de manter o decúbito elevado para prevenção de pneumonia associada à ventilação mecânica após ação educativa sobre a importância dessa intervenção.

Aprendizagem é um processo complexo, que envolve muitas variáveis e está sujeito à influência de fatores internos, individuais e externos $^{10}$. Também pode ser entendida com o conceito de que os seres adquirem novos conhecimentos e competências, provocando modificações de forma relativamente durável de seus comportamentos. Dessa forma, apesar de ser um evento interno e pessoal, só as ações manifestas permitem concluir se houve ou não aprendizado. Neste estudo, apesar de a ação educativa ter sido planejada, individualizada, com o uso de recursos visuais e no contexto do ambiente de trabalho, não foi possível manter o comportamento esperado, de posicionar a cabeceira do leito elevada, por mais de dois meses. Ao ser retirado o cartaz com as imagens da relação da posição do paciente no leito com a aspiração pulmonar, houve queda na angulação do decúbito, ressaltando a importância do reforço positivo na manutenção do comportamento desejado ${ }^{11}$.

A patogênese da pneumonia associada à ventilação mecânica é multifatorial. $\mathrm{O}$ uso da ventilação mecânica invasiva associada à aspiração de secreção orofaríngea colonizada para os pulmões são fatores importantes para o desenvolvimento dessa complicação, associada à vulnerabilidade do paciente grave por desnutrição e alterações no sistema imunológico ${ }^{12}$. O decúbito elevado foi introduzido na prática clínica para prevenir a aspiração pulmonar de micro-organismos presentes na orofaringe. Na posição de decúbito elevado, o refluxo do conteúdo gástrico é contrabalanceado pela ação da gravidade e auxilia os movimentos peristálticos e o esvaziamento gástrico. Além disso, é possível que a posição elevada melhore a ventilação e o recrutamento alveolar nos lobos

Tabela 2: Caracterização dos pacientes admitidos na Unidade de Terapia Intensiva, Londrina, Paraná, 2010

\begin{tabular}{|l|c|c|c|}
\hline \multirow{2}{*}{ Características dos pacientes } & Pré-intervenção & Pós-intervenção & Valor p \\
\hline Gênero masculino* & $\mathbf{( n = 2 5 )}$ & $\mathbf{( n = 1 4 5 )}$ & 0,079 \\
\hline Idade (anos)† & $11(44,0 \%)$ & $89(61,4 \%)$ & 0,173 \\
\hline APACHE II† & $62,64 \pm 18,32$ & $56,57 \pm 20,82$ & 0,395 \\
\hline SOFA† & $20,80 \pm 8,95$ & $19,09 \pm 9,26$ & 0,838 \\
\hline Ventilação mecânica na admissão* & $6,60 \pm 3,66$ & $6,43 \pm 3,76$ & 0,127 \\
\hline Ventilação mecânica na UTI* & $13(52,0 \%)$ & $96(66,2 \%)$ & 0,104 \\
\hline Dieta enteral* & $14(56,0 \%)$ & $103(71,0 \%)$ & 0,231 \\
\hline Dias de ventilação mecânica† & $13(52,0 \%)$ & $90(62,1 \%)$ & 0,941 \\
\hline Dias na UTI† & $7,48 \pm 11,76$ & $7,64 \pm 10,35$ & 0,825 \\
\hline PAV* & $9,56 \pm 11,05$ & $10,04 \pm 10,04$ & 0,148 \\
\hline Mortalidade* $^{*}$ & $11(44,0 \%)$ & $45(31,0 \%)$ & 0,396 \\
\hline
\end{tabular}

*Valores expressos em número e porcentagens, valor p pelo teste exato de Fisher; †Valores expressos em média e desvio padrão, valor de p pelo teste $t$ de Student; APACHE II: Acute Physiology and Chronic Health Evaluation; SOFA: Sequential Organ Failure Assessment; PAV: Pneumonia Associada à Ventilação Mecânica; UTI: Unidade de Terapia Intensiva. 
pulmonares inferiores e que esse efeito tenha um papel complementar na prevenção da infecção pulmonar ${ }^{13}$.

A implementação de diretrizes para prevenção de infecções relacionadas à assistência à saúde pode ser facilitada quando proposto um conjunto de medidas simultâneas para atingir esse objetivo. Essas medidas são conhecidas como bundles de cuidados ou pacotes de intervenções. Tal abordagem tem o objetivo de aumentar a adesão às recomendações e reduzir a incidência de infecções relacionadas à assistência à saúde.

Recentemente, foi proposto um pacote de cuidados para a prevenção de pneumonia associada à ventilação mecânica, com aplicação de metodologia baseada em evidências e análise multicritérios no processo de decisão ${ }^{14}$. A educação e o treinamento dos profissionais de saúde são fatores-chave para aumentar a adesão às diretrizes. Nesse processo de cuidados é de vital importância a participação de todos os profissionais envolvidos no cuidado ao paciente.

A mudança efetiva de um processo de trabalho envolve não somente treinamento e educação constantes como também auditoria com geração de dados sobre adesão em tempo real e um processo de feedback. Os profissionais envolvidos no cuidado do paciente devem ter acesso rápido a esse feedback sobre sua performance no cuidado ao paciente e qual o impacto na qualidade do cuidado prestado. Dessa forma, é possível atingir mudanças duradouras nos processos de cuidados aos pacientes.

No presente estudo foi demonstrado aumento da adesão à recomendação de manter o decúbito elevado e concomitante redução na frequência de pneumonia, porém esses achados foram transitórios. A observação de perda progressiva da adesão a essa recomendação já foi descrita por outros autores ${ }^{15}$, que observaram redução da angulação do decúbito de 28 para 22 graus em uma semana.

No presente estudo não foi detectada redução na frequência de pneumonia associada à ventilação mecânica, apesar do aumento da média da angulação do decúbito, porém o número de eventos observados pode não ter conferido poder ao estudo para detectar tal diferença. Por outro lado, estudos já demonstraram que as posições prona ${ }^{16}$ ou horizontal lateral com orientação da traqueia abaixo da linha horizontal ${ }^{17}$ apresentam como vantagem o favorecimento da drenagem da secreção supraglótica, acima do cuff do tubo traqueal, evitando a aspiração pulmonar e prevenindo infecções pulmonares.
O cenário onde o estudo foi desenvolvido constitui-se em uma unidade com pacientes de alta gravidade, refletido pela média de APACHE II acima de 19, e se associa com uma mortalidade esperada em torno de $30 \%$. A frequência de pneumonia foi alta no período de estudo, provavelmente pelo uso frequente de ventilação mecânica associada à administração de nutrição enteral. Em ambientes com pacientes de alta gravidade, complexidade e portadores de diversos fatores de risco para infecção, o reconhecimento da potencialidade de ocorrência de erros durante o cuidado e a elaboração de estratégias para redução dessas falhas é fundamental para aumentar a segurança do paciente ${ }^{18}$. Mudanças na percepção e na cultura da segurança no cuidado aos pacientes hospitalizados representam fatores-chave para sucesso na prevenção e no controle das infecções relacionadas à assistência à saúde, assim como propostas de educação e treinamento em serviço auxiliam a atingir tais objetivos.

Existem limitações que devem ser consideradas em relação ao presente estudo. É um estudo de centro único que avaliou exclusivamente uma das medidas propostas pelo conjunto de ações recomendadas para redução da incidência de pneumonia associada à ventilação mecânica. $\mathrm{O}$ foco do estudo foi o treinamento da equipe de saúde e o efeito da ação educativa no processo de trabalho. Portanto, o estudo não foi delineado para avaliar incidência de pneumonia, tendo sido realizado um período curto de observação clínica em um número reduzido de pacientes, o que não permite conclusões sobre o efeito dessa ação na incidência de pneumonia. A ação educativa proposta neste estudo é uma das ações que devem ser tomadas no processo complexo de prevenção da pneumonia adquirida em UTI, mas é um passo inicial crítico.

Em conclusão, o presente estudo constatou que a ação educativa teve efeito positivo transitório na adesão à recomendação de manutenção do decúbito elevado para prevenção da pneumonia associada à ventilação mecânica. A falta de persistência dos nossos resultados de adesão em manter decúbito elevado provavelmente reflete a ausência de programas de auditoria e feedback associados ao programa de treinamento implementado. Dessa forma, evidencia-se a necessidade de implantação de programas de treinamento associados a um sistema de auditoria e feedback para avaliação de resultados.

\section{REFERÊNCIAS}

1. Russell CJ, Shiroishi MS, Siantz E, Wu BW, Patino CM. The use of inhaled antibiotic therapy in the treatment of ventilator-associated pneumonia and tracheobronchitis: a systematic review.BMC Pulm Med. 2016;16:40.

http://dx.doi.org/10.1186/s12890-016-0202-8

2. Bassi GL, Ferrer M, Marti JD, Comaru T, Torres A. Ventilatorassociated pneumonia. Semin Respir Crit Care Med. 2014;35(4):469-81

http://dx.doi.org/10.1055/s-0034-1384752
3. Keyt H, Faverio P, Restrepo MI. Prevention of ventilatorassociated pneumonia in the intensive care unit: a review of the clinically relevant recent advancements. Indian $\mathrm{J}$ Med Res. 2014;139(6):814-21.

4. Suhas $P$, Kundra P, Cherian A. Polyurethane cuffed versus conventional endotracheal tubes: Effect on ventilator-associated pneumonia rates and length of Intensive Care Unit stay. Indian J Anaesth. 2016;60(3):163-7. http://dx.doi.org/10.4103/0019-5049.177871 
5. Wang L1, Li X, Yang Z, Tang X, Yuan Q, Deng L, et al. Semirecumbent position versus supine position for the prevention of ventilator-associated pneumonia in adults requiring mechanical ventilation. Cochrane Database Syst Rev. 2016;1:CD009946. http://dx.doi.org/10.1002/14651858.CD009946.pub2

6. Ferreira CR, Souza DF, Cunha TM, Tavares M, Reis SS, Pedroso $\mathrm{RS}$, et al. The effectiveness of a bundle in the prevention of ventilator-associated pneumonia. Braz $J$ Infect Dis. 2016;20(3):267-71 http://dx.doi.org/10.1016/j.bjid.2016.03.004

7. Vasilevskis EE, Pandharipande PP, Graves AJ, Shintani A, Tsuruta R, Ely EW, et al. Validity of a Modified Sequential Organ Failure Assessment Score Using the Richmond Agitation-Sedation Scale. Crit Care Med. 2016;44(1):138-46. http://dx.doi.org/10.1097/CCM.0000000000001375

8. Hashmi M, Asghar A, Shamim F, Khan FH. Validation of acute physiologic and chronic health evaluation II scoring system software developed at The Aga Khan University, Pakistan. Saudi J Anaesth. 2016;10(1):45-9. http://dx.doi.org/10.4103/1658-354X.169474

9. McMullen KM, Boyer AF, Schoenberg N, Babcock HM, Micek ST, Kollef $\mathrm{MH}$. Surveillance versus clinical adjudication: differences persist with new ventilator-associated event definition. Am J Infect Control. 2015;43(6):589-91. http://dx.doi.org/10.1016/j.ajic.2015.03.004

10. Ellery AEL, Bosi MLM, Loiola FA. Integração ensino, pesquisa e serviços em saúde: antecedentes, estratégias e iniciativas. Saude Soc. 2013;22(1):187-98. http://dx.doi.org/10.1590/S0104-12902013000100017

11. Beckert ME. Correspondência verbal/não verbal: pesquisa básica e aplicações na clínica. In: Abreu-Rodrigues J, Ribeiro
RM. Análise do comportamento: pesquisa, teoria e aplicação. Porto Alegre: Artmed; 2005; p.229-44

12. Mietto C, Pinciroli R, Patel N, Berra L. Ventilator associated pneumonia: evolving definitions and preventive strategies. Respir Care. 2013;58(6):990-1007. http://dx.doi.org/10.4187/respcare.02380

13. Bassi GL, Torres A. Ventilator-associated pneumonia: role of positioning. Curr Opin Crit Care. 2011:17(1):57-63. http://dx.doi.org/10.1097/MCC.0b013e3283428b31

14. Rello J, Lode H, Cornaglia G, Masterton R, VAP Care Bundle Contributors. A European care bundle for prevention of ventilatorassociated pneumonia. Intensive Care Med. 2010;36(5):773-80. http://dx.doi.org/10.1007/s00134-010-1841-5

15. Van Nieuwenhoven CA, Vandenbroucke-Grauls $\mathrm{C}$, van Tiel FH, Joore HC, van Schijndel RJ, van der Tweel I, et al. Feasibility and effects of the semi recumbent position to prevent ventilator associated pneumonia: a randomized study. Crit Care Med. 2006;34(2):396-402

16. Sud S, Friedrich JO, Taccone P, Polli F, Adhikari NK, Latini R, et al. Prone ventilation reduces mortality in patients with acute respiratory failure and severe hypoxemia: systematic review and meta-analysis. Intensive Care Med. 2010;36(4):585-99. http://dx.doi.org/10.1007/s00134-009-1748-1

17. Mauri T, Berra L, Kumwilaisak K, Pivi S, Ufberg JW, Kueppers F, et al. Lateral-horizontal patient position and horizontal orientation of the endotracheal tube to prevent aspiration in adult surgical intensive care unit patients: a feasibility study. Respir Care. 2010;55(3):294-302.

18. Blot S, Lisboa T, Angles R, Rello J. Prevention of VAP: Is zero rate possible? Clin Chest Med. 2011:32(3):591-9. http://dx.doi.org/10.1016/j.ccm.2011.05.008 